\title{
Automatic Detection of Stellate Lesions in Digital Mammograms Using Multi-scale SIFT
}

\author{
A. Hikmat ${ }^{1}$, K. Afdel $^{2}$ and I. Bakkouri ${ }^{2}$ \\ 1. Laboratory of the Aquatic Systems: Marine and Continental Environment, Faculty of Science, Ibn Zohr, University, BP 8106, \\ Agadir 80000, Morocco \\ 2. LABSIV, Department of Computer Science, Faculty of Science, Ibn Zohr University, Agadir, BP 8106, Agadir 80000, Morocco
}

\begin{abstract}
This paper presents a novel automatic mammography recognition approach used to develop computer-aided diagnostic systems that require a robust method to assist the radiologist in identifying and recognizing speculations from a multitude of lines corresponding to the normal fibrous breast tissue. Following this rationale, this paper introduces a novel approach for detecting the speculated lesions in digital mammograms based on multi-scale SIFT (scale-invariant feature transform) orientations. The proposed method starts by estimating a set of key points that best represent the image mammography in a scale space. We then benefit from SIFT algorithm to locally characterize each key point by assigning a consistent orientation. Thereafter, a set of three features are extracted for each pixel in the image mammogram based on these orientations. The extracted features are fed into BDT (binary decision tree) in order to perform per pixel classification and decide whether the pixel is normal or abnormal. We evaluate the proposed system on BCDR (breast cancer digital repository) database and the experimental results show that our method is accurate with 97.95\% recognition rate, while it is robust to illumination changes, rotation and scale variations.
\end{abstract}

Key words: Digital mammograms, speculated lesions, SIFT orientation, BDT.

\section{Introduction}

The breast cancer is a one of the famous cancers that heavily infects women worldwide. It is also the most significant causes of increased women fatality rate. The quick spread of breast cancer in developing countries can be attributed to the longer life expectancy, the increase and intensification of urbanization, and the lifestyles adoption. In fact, even though the prevention is mandatory in these countries, it only reduces the risk of injuring by breast cancer but not eliminating it. Furthermore, early detection of this type of tumors is not easy, and most detection occurs at very late stages. Mammography, which is a breast image taken with a special X-ray, has demonstrated, by far, the best diagnostic tool for early detection of breast cancer symptoms. However, there are some factors that make the detection of stellate lesions very difficult and

Corresponding author: Abdessamad HIKMAT, PhD, research fields: Marine and Continental Environment. challenging task, such as: variation in shape, appearance and density of breast tissue, accompanied noise during cancer screening, and low contrast digital mammograms. Consequently, the probability of getting false negative and false positive results is likely to increase. The person's age can also impact the detection results; since young women with denser breast tissue are more likely to get false results than old women. All these factors bring the necessity of using more sophisticated and costly techniques such as ultrasound, MRI (magnetic resonance imaging), and sometimes biopsy to verify the presence or absence of a malignant stellate mass.

Several mammographic pattern recognition methods have been used to distinguish speculated linear structures from normal ones of the breast. These approaches include the shapes with their margins analysis which are often seen in the Circumscribed masses but are not typical for stellate lesions detection. In the normal linear structures, the lesions radiates in 
a similar direction which tends toward the nipple with parallel orientation (Fig. 1a). The breast tissue histology can be considered a branching system of ducts which connect repeatedly giving rise to the terminal duct lobular unit. It is composed of large and fibrous tissue in different proportions (Fig. 1b) [1]. By contrast, the speculated lesions described as irregular shape and stellate margins with a central mass, less than $1 \mathrm{~cm}$ in diameter which aligned in all directions (Fig. 2a). Histologically, the abnormal regions arise from the epithelial tissue of the breast. The epithelial entity consists of the cells that line the terminal duct lobular unit. It exhibits the highly flexible fibrous spicules which radiate in the different directions (Fig. 2b) [1].

Now, It is easy to differentiate normal breast parenchymal patterns from speculated ones and identify spatial positions of the lines by capturing the orientation features that characterize the stellate appearance of mass (Figs. 1c and 2c).

The proposed method starts by estimating a set of key points that best represent the image mammography in a scale space. We then benefit from SIFT (scale-invariant feature transform) algorithm [2] to locally characterize each key point by assigning a consistent orientation. Thereafter, a set of three features are extracted for each pixel in the image mammogram based on these orientations. The extracted features are fed into BDT (binary decision tree) in order to decide whether the corresponding pixel is normal or abnormal. The proposed system is evaluated on BCDR (breast cancer digital repository) database and the experimental results show that our method is accurate with $97.95 \%$ recognition rate, while it is robust to illumination, rotation and scale variance.

The remainder of this article is organized as follows: in Section 2, we review some related works in the area of breast cancer detection. In Section 3, we detail our
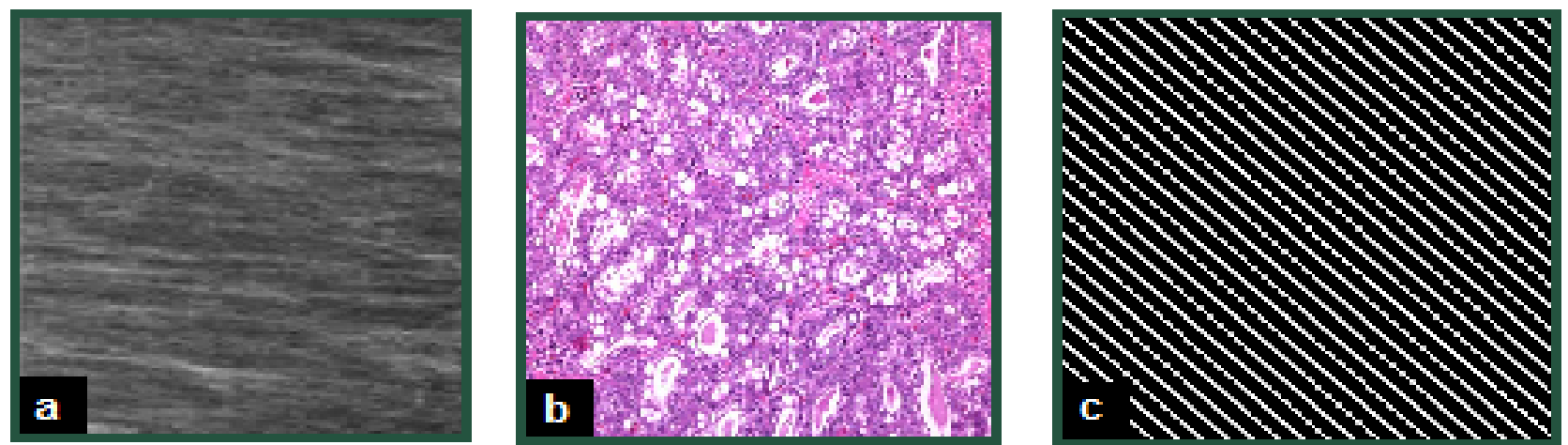

Fig. 1 Examples of normal radiological structure in standard mammography: (a) normal linear structure; (b) normal breast tissue histology; (c) simulation image of normal lesion.
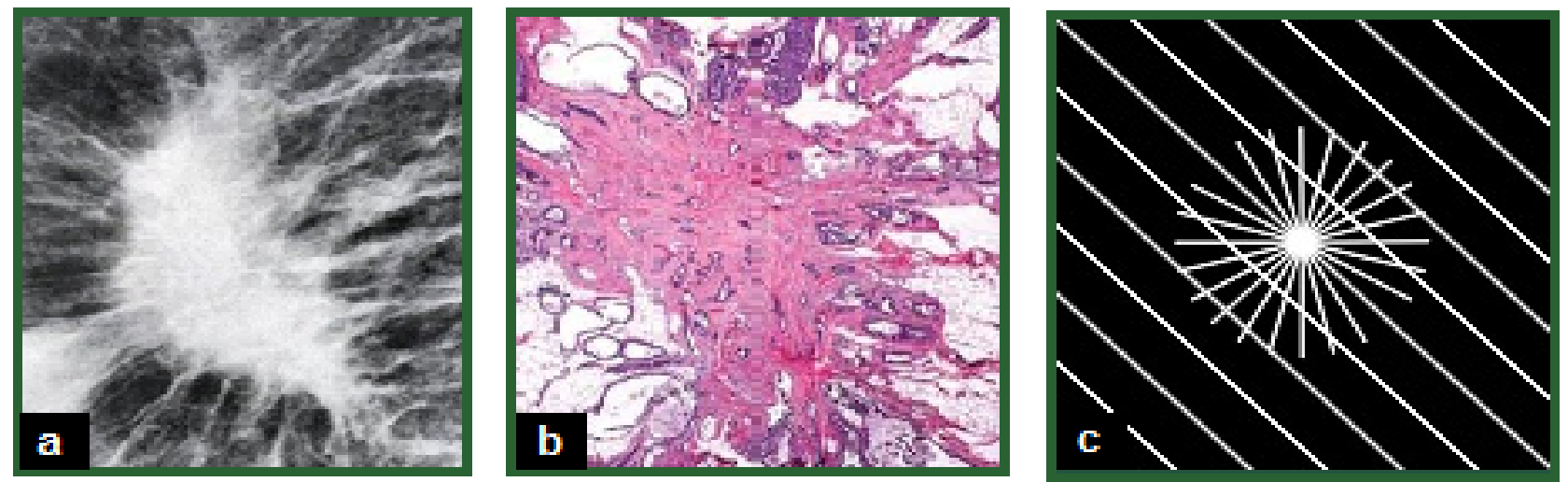

Fig. 2 Examples of abnormal radiological structure in standard mammography: (a) speculated linear structure; (b) abnormal breast tissue histology; (c) simulation image of stellate lesion. 
approach for stellate lesions detection in digital mammograms. In Section 4, we present our experimental results on two popular benchmark datasets. The last Section 5 concludes the paper.

\section{Related Work}

There have been a plethora of approaches for stellar lesions characterization proposed in the literature. Some of these approaches attempt to detect the central mass of the stellar lesions, while other approaches concentrate on the detection of spicules [3, 4]. However, there are some factors that generally make the implementation of an accurate system to detect these opacities very difficult, such as: (1) the very low contrast of the majority of mammography images, (2) significant variability in the density of breast tissue in digital mammogram which typically depends on the age of the patients, and (3) the plentiful textured structure of the breast tissue.

In particular, Ng and Bishof [5] proposed a technique to detect the central stellar mass lesions using the so-called form-fitting technique (template-matching), where a combination of textural analysis and laws mask is used for architectural distortion localization. In Ref. [6], Kegelmeyer et al. developed a detection method based on the local analysis of contours orientations with Hough transform. Hough transform was also used in Ref. [7] to detect the stellar lesions. In Ref. [8], the independent component analysis with neural networks is used to detect and classify the malignant and benign lesions. Though their method achieved comparatively great recognition results (accuracy of 97.3\%), it is highly sensitive to scale and rotation variance. Ballerini and Franzen [9] applied an optimal filter on the breast image to extract the characterization parameters. Their method was able to detect the circumscribed masses as well as stellar lesions. The skeleton lines for the detection of speculations are applied in Ref. [10]. The directional recursive median filter was proposed in Ref. [11] for the detection of the central stellar mass lesions. The method proposed in Ref. [12] takes into account both the direction of spicules and their density, namely, they first applied an open operation to increase the shadows of spicules, and then they established a pixel orientation map to determine two parameters using the concentration expression. On the other hand, Sampat et al. [13] developed an iris filter that applies the image gradient to enhance the brightness of the lesions.

Other methods, such as Refs. [14, 15], used what is so called the wavelet transform to classify masses from descriptive parameters of shape. It has been also used in Ref. [3] to decompose the mammography image into multiple resolutions, and for each resolution, they extract the directional information to detect the speculated lesion. However, the wavelet transform decomposition-based methods are not translation invariant and can produce a noticeable amount of quantization noise which can greatly affect the quality of the image [16]. Furthermore, the traditional way, by which the gradient directions are computed, is not robust to scale change and requires a long computational time.

Although the above-mentioned methods have achieved great results in the detection of speculated lesions, still, there are some limitations that generally affect their performances, which are: (i) the use of a user-defined threshold in the preprocessing stage will result in a loss of substantial information contained in the original mammography image; (ii) most of the methods in the literature are not robust to scale variance, which make their performances limited to the size of the stellate lesion; (iii) the use of contrast enhancement tools to increase the mammography visibility will have a negative impact on the binarization of the image, thus, on the detection of stellar lesions; (iv) the oriented contours map, resulted using Gabor filters, is limited to a few specific numbers of directions, which make the statistical computation of their dispersion inaccurate.

Addressing the above limitations, we alternatively present in this paper a novel method for detection of 
speculated lesions in digital mammograms. In the proposed approach, the notion of multi-scale SIFT orientations is used to efficiently characterize the stellate lesions.

\section{Proposed Method}

Most of the approaches found in the literature seek to characterize the stellar lesions by detecting the orientations of their structures, each of which uses a specific technique to determine these orientations, such as Gabor filters, contrast enhancement, thresholding, and so on. In spite of that, these approaches are limited in detecting only a few specific orientations, which make them inaccurate in describing the spread of the suspicious lesions. In addition, they are very sensitive to the common image transformations.

Our main contribution in this paper is to use the SIFT orientations [2] to extract the characteristics of stellar lesions, which make them invariant to the image translation, scaling and rotation; and partially insensitive to the light conditions and 3D projection or local geometric distortions. The proposed approach will provide a rich and robust representative orientations map, which eventually will facilitate the statistical study.

Fig. 3 shows the proposed method for stellar lesions detection. It encompasses two main steps. The first step, extraction of pixel characteristics, aims at computing the statistical features of each pixel in the image by using the SIFT orientation map of stellate lesions. The last step, classification, performs the per-pixel classification using a combination of BDT classifier and a set of statistical parameters computed from the orientation map of these suspicious regions.

\subsection{Extraction of Pixel Characteristics}

Once the noise is removed and the mammography image is enhanced, the next step is to characterize the speculated lesions. Throughout this step, we first start by detecting the set of stable key points that best represent the image mammography in a scale space. We then locally characterize each key point using SIFT orientations. Lastly, we compute a set of three statistical features for each pixel in the mammography

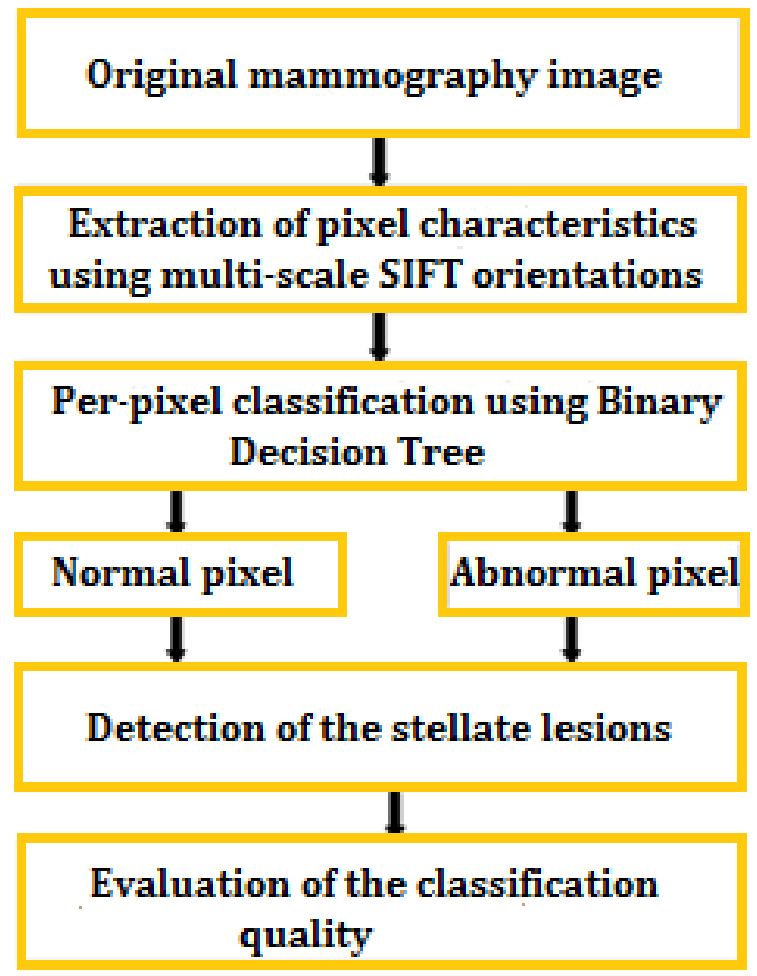

Fig. 3 General overview of the proposed approach. 
image based on these orientations.

\subsection{Extraction of SIFT Key Points}

One of the major challenges in the detection of stellar lesions is the variation of their size, which ranges from a few millimeters to several centimeters in diameter. Indeed, Ng and Bishof [5] found that the stellar form of the suspicious lesions is preserved when passing from the high resolution to small resolution. In our approach, we use the DoG (Difference of Gaussian), proposed by Lowe [2], to decompose the image $I$ into a low resolution image pyramid, so that stellar lesions detection proceeds successfully despite changes in scale, illumination, noise and distortion. The pyramid is typically a multi-scale representation, where the image is decomposed by successively applying the low-pass Gaussian filters with sampling. Note that the lesions are preserved during this multi-spectral representation.

In order to detect the SIFT key points, we first build a scale space using the DoG function:

$$
\begin{gathered}
\operatorname{DoG}(x, y, \sigma)=[G(x, y, k \sigma)-G(x, y, \sigma)] \\
\quad * I(x, y)
\end{gathered}
$$

where:

$$
G(x, y, \sigma)=\frac{1}{2 \pi \sigma^{2}} e^{\frac{\left(x^{2}+y^{2}\right)}{2 \sigma^{2}}}
$$

The function $G(x, y, \sigma)$ (Eq. (2)) is the Gaussian
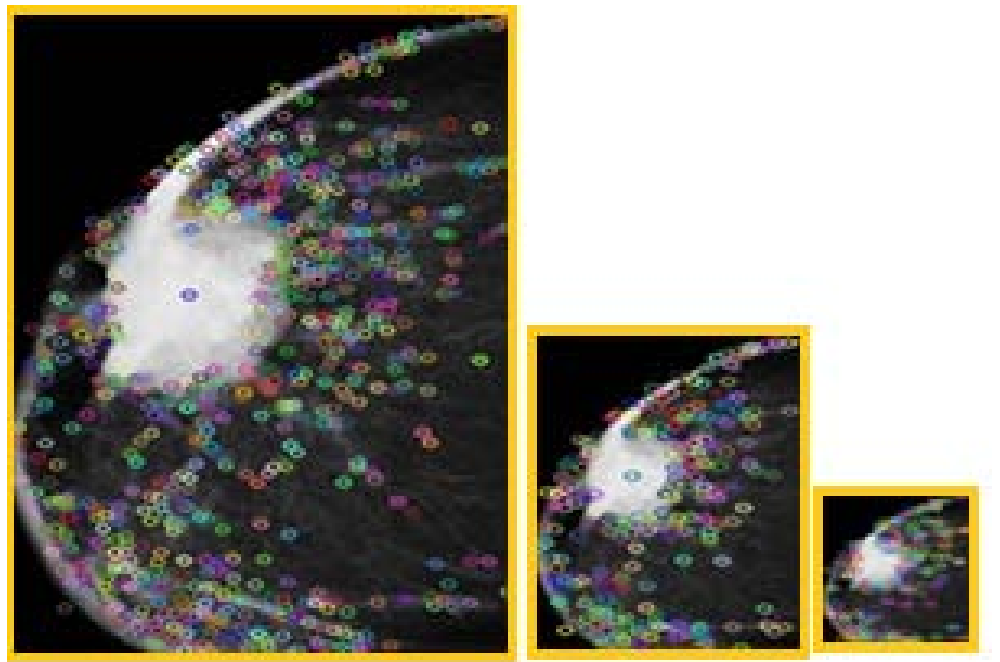

Fig. 4 Key points identification for a sample image with three resolutions using DoG function.

. Key points identification for a sample image with three resolutions using DoG function. kernel, $k$ represents the scale factor, and $I(x, y)$ denotes the mammography image pixel.

Thereafter, we compare the intensity of each pixel in the scale space images to its eight neighbors at the same scale, and to nine corresponding neighboring pixels in each of the neighboring scales. We then keep the pixels that represent the maximum or the minimum as stable key points $\{p=(x, y)\}$, and the remaining points will be rejected. Note that this extracted set of key points includes both the key points located on the mammography image regions and contours. Fig. 4 shows the extracted key points from a sample mammography image with multiple resolutions. We observe that these key points are situated on the lesion as well as on the normal breast structure.

\subsection{Computation of SIFT Orientations}

In this subsection, we assign a consistent orientation $\varphi$ to each extracted key point in the mammography image $I$. Namely, as explained in Ref. [2], the gradient orientation $\theta$ is firstly computed for each image sample $I(x, y)$, at a given scale, as:

$$
\theta=\tan ^{-1}\left[\frac{I(x, y+1)-I(x, y-1)}{I(x+1, y)-I(x-1, y)}\right]
$$

Then, an orientation histogram is built from the gradient orientations at all sample points within a circular window around the corresponding key point. 
The orientation histogram has 36 bins covering the 360 degree range of orientations. We therefore detect the highest local peak in the orientation histogram as dominant directions of local gradients. The orientation corresponding to this high peak is eventually treated as the local orientation $\varphi$ of its respective key point. By repeating this procedure for each extracted key point in the image $I$, we will obtain an orientation map that characterize the stellate lesions.

Notice that stellar lesion differs to the mammary structure of normal breast ducts. This structure tends to radiate from the nipple to the chest wall. However, the tumor appears as an irregular center surrounded by radiated lines in all directions and drowned in the breast structure. Specifically discussing, the gradient orientations $\varphi$ of key points in normal regions are pointed from the nipple toward the chest wall, whilst, the gradient orientations $\varphi$ of key points located in stellar lesion tend to spread in all directions, as visualized in Fig. 5b. These dispersed directions are the key-characteristic for detecting the presence or absence of stellate lesions. Consequently, the histogram of the gradient orientations $\varphi$ corresponding to the area of the stellate lesion will be flat and homogeneous, whereas, it will have a very pronounced peak in case of normal breast tissue region (see Figs. 5 and 6 for example).

\subsection{Extraction of Statistical Features}

In order to distinguish between stellar lesions and the normal breast tissue, we place at each pixel $(x, y)$ in digital mammogram $I$ a sliding window of $16 \times 16$
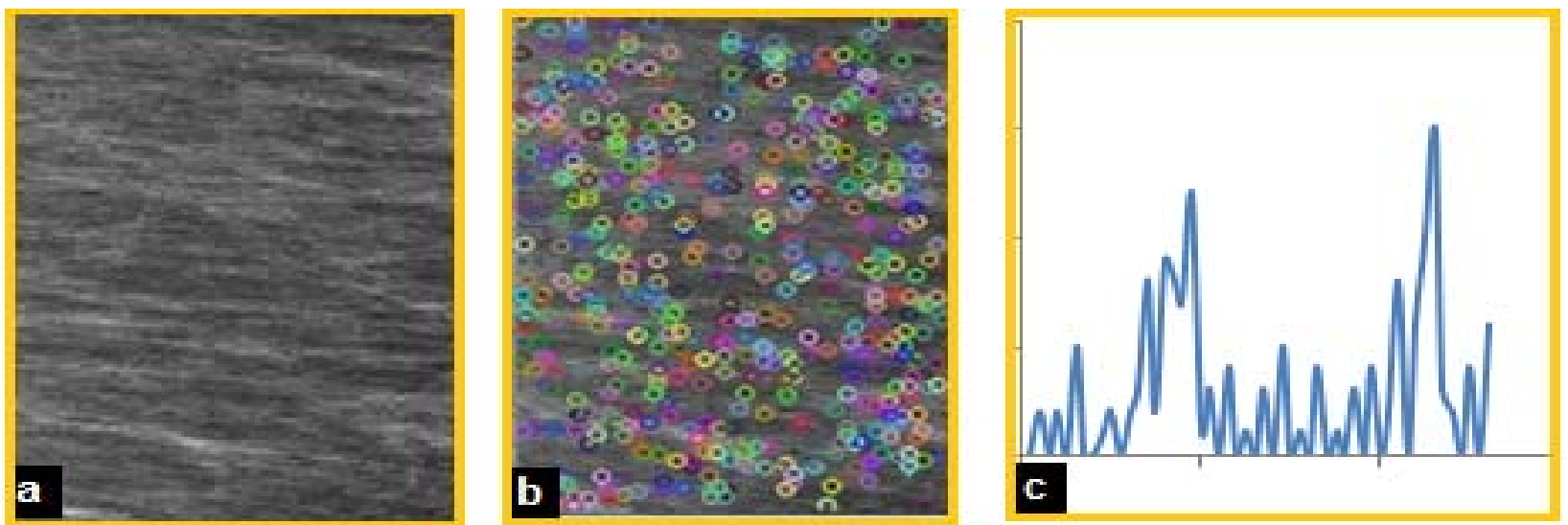

Fig. 5 Orientation histogram corresponding to the normal breast ducts: (a) example of normal breast tissue; (b) detection of key points; (c) the histogram of gradient orientations.
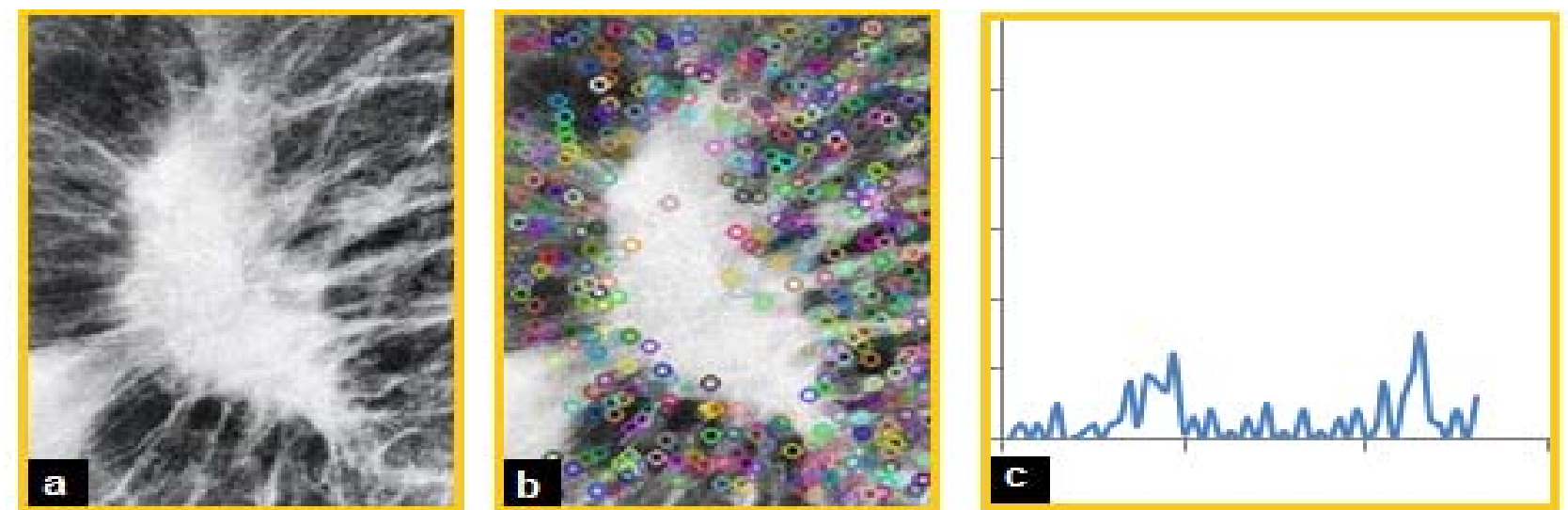

Fig. 6 Orientation histogram corresponding to the spiculated region: (a) example of stellar lesion; (b) extraction of key points; (c) the histogram of gradient orientations. 
pixels in size, centred on the pixel $(x, y)$. Then, three types of statistical features characterizing this pixel are computed based on the set of gradient orientations $\varphi$ that are exclusively located within the $16 \times 16$ sliding window. These three features are: mean of the gradient directions, standard deviation of gradient directions, and standard deviation of the gradient orientations histogram.

\subsubsection{Mean of the Gradient Directions}

Let $(x, y)$ be the coordinates of a pixel in mammography image $I, \partial S_{(x, y)}$ be the set of key points $\{p=(m, n)\}$ that are located within the $16 \times$ 16 sliding window, and $\varphi(m, n)$ be the gradient orientation corresponding to $p=(m, n)$. The average gradient orientation $\overline{\varphi(x, y)}$ in the pixel $(x, y)$ is defined as:

$$
\overline{\varphi(x, y)}=\frac{1}{\left|\partial S_{(x, y)}\right|} \sum_{p=(m, n) \in \partial S_{(x, y)}} \varphi(m, n)
$$

\subsubsection{Standard Deviation of Gradient Directionss}

The standard deviation of gradient directions $\sigma(x, y)$ in the pixel $(x, y)$ can be mathematically expressed as: (5)

$$
=\sqrt{\frac{1}{\left|\partial S_{(x, y)}\right|-1} \sum_{p=(m, n) \in \partial S_{(x, y)}}(\varphi(m, n)-\overline{\varphi(x, y)})^{2}}
$$

3.4.3 Standard Deviation of the Gradient Orientations Histogram

To compute this feature, we first built a histogram of gradient orientations $\varphi(m, n)$ particularly belonging to the set $\partial S_{(x, y)}$. Let $\operatorname{Hist}_{(x, y)}(i)\{i=0,1, \ldots, q\}$ be this histogram. In our experiments, we set $q=255$ bins. We then compute the average value of this histogram as:

$$
\overline{\operatorname{Hist}_{(x, y)}}=\frac{1}{q+1} \sum_{i=0}^{q} \operatorname{Hist}_{(x, y)}(i)
$$

The standard deviation of this histogram gives us the correlation between the value of gradient orientations $\varphi(m, n)$ and the number of key points that have the same gradient direction:

$$
\sigma_{\text {Hist }}(x, y)=\sqrt{\frac{1}{q} \sum_{i=0}^{q}\left(\operatorname{Hist}_{(x, y)}(i)-\overline{\operatorname{Hist}_{(x, y)}}\right)^{2}}
$$

\subsection{Classification}

In order to detect the stellate lesions in digital mammograms, we perform the per-pixel classification algorithm using the well-known BDT [17]. Namely, for each pixel $(x, y)$ in digital mammogram, we form a feature vector from the three statistical features $\left(\overline{\varphi(x, y)}, \sigma(x, y)\right.$ and $\left.\sigma_{\text {Hist }}(x, y)\right)$ computed in Section 3.4.1. Then, we fed this feature vector in BDT classifier to decide whether the corresponding pixel is normal or abnormal. The objective behind using BDT in our classification stage is that, it is simple, easy to implement and computationally less expansive, also it can handle both numeric values and categories.

\section{Experimental Results}

The purpose of medical classification of diseases is to gather the cases that have some biological similarities and that are likely to share some histopathological factors. Identifying such classes is important because it allows, on one hand, understanding the disease process, on the other hand, establishing the appropriate therapeutic approach. It also permits to identify the global prognosis of the disease. In what follows, we present our experimental results to evaluate the performances of the proposed speculated lesions detection system.

\subsection{Datasets and Experimental Setup}

In order to evaluate the performances of the proposed method, we have used the BCDR [18]. It is a public dataset of mammography images. BCDR is the first Portuguese digital mammograms. It has at present a total of 1,010 cases including digital content (3,703 digitized film mammography images) and associated metadata. Precise segmentations of identified lesions 
(manual contours made by medical specialists) are also provided. In total 795 lesions were segmented in MLO and CC images to produce a total of 1,493 segmentations, which are included: masses $=639$, microcalcifications $=341$, calcifications $=145$, stromal distortions $=102$, architectural distortions $=$ 66 , and axillary adenopathy $=2$. Currently, the BCDR contains cases of 1,734 patients with mammography and ultrasound images, clinical history, lesion segmentation and selected pre-computed image-based descriptors. Patient cases are BIRADS (breast imaging reporting and data system) [1] classified and annotated by specialized radiologists. In this work, we have selected 4 cases from digital BIRADS 1, 328 cases from digital BIRADS 2, 80 cases from digital BIRADS 3 and 176 cases from digital BIRADS 4.

As for learning the BDT classifier, we have used 294 mammography images from BCDR dataset as a training set. This set includes 166 normal images and 128 images with anomalies. As we mentioned above, the anomalies in the training set have been identified by expert radiologists and interpreted by BIRADS system. For testing stage, we use 294 mammography images, 166 of which are normal and the remaining 128 images contain peculated lesions.

\subsection{Quantitative Evaluation}

The classification results of speculated lesions obtained by our method are reported in Table 1 . As it can be seen, the proposed system demonstrates its ability in distinguishing between benign and malignant mammography images, and the classification results are generally quite satisfactory, with $97.95 \%$ in average. Table 2 summarizes the qualitative evaluation results of our detection approach in terms of sensitivity rate, specificity rate, classification rate and error rate. Furthermore, the performance of our method is also evaluated using ROC (receiver operating characteristic) curve $[19,20]$, as it can be seen in Fig. 7. We empirically observe that the results are well encouraging, with unnoticeable performance loss.

\subsection{Qualitative Evaluation}

To consolidate our experiments, we have also illustrated in Fig. 8 the detection states of stellate lesions for some image samples from BCDR dataset using the proposed method. As visualized in Fig. 8, we can clearly claim that our approach is qualitatively able to accurately detect stellate lesions despite change in image contrast, scale, illumination and distortion.

\subsection{Comparison with State-of-the-Art Methods}

A comparative study between our approach and other detection methods, found in the literature, is illustrated in Table 3. It is clearly visible that our method outperforms all the compared baselines, and is even better than neural networks-based approaches. This can be attributed to the fact that SIFT orientations are more representative, robust and compact. These advantages of SIFT orientations have been verified practically for spiculated lesions detection, which permit to achieve a high detection rate of $97.95 \%$.

Table 1 Confusion matrix of breast lesions classification given by our proposed model.

\begin{tabular}{lll}
\hline & Abnormal & Normal \\
\hline Abnormal & 164 & 2 \\
Normal & 4 & 124 \\
\hline
\end{tabular}

Table 2 Qualitative evaluation results of the proposed method.

\begin{tabular}{lllll}
\hline & Sensitivity rate & Specificity rate & Classification rate & Error rate \\
\hline Results & $98.79 \%$ & $96.87 \%$ & $97.95 \%$ & $2.05 \%$ \\
\hline
\end{tabular}




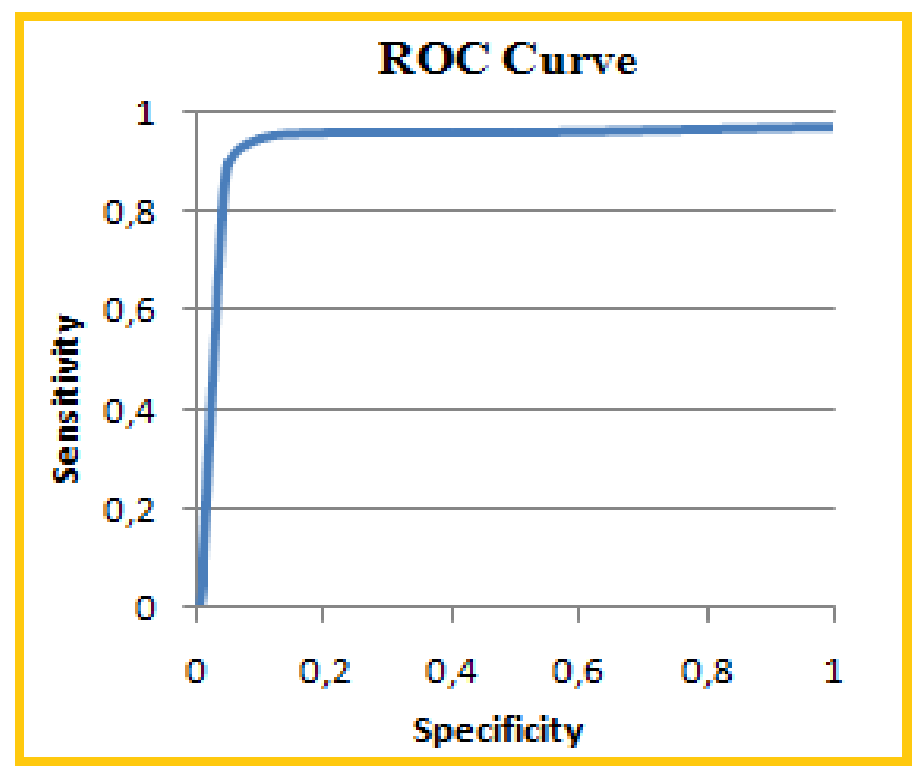

Fig. 7 ROC curve for the detection of stellar lesions.
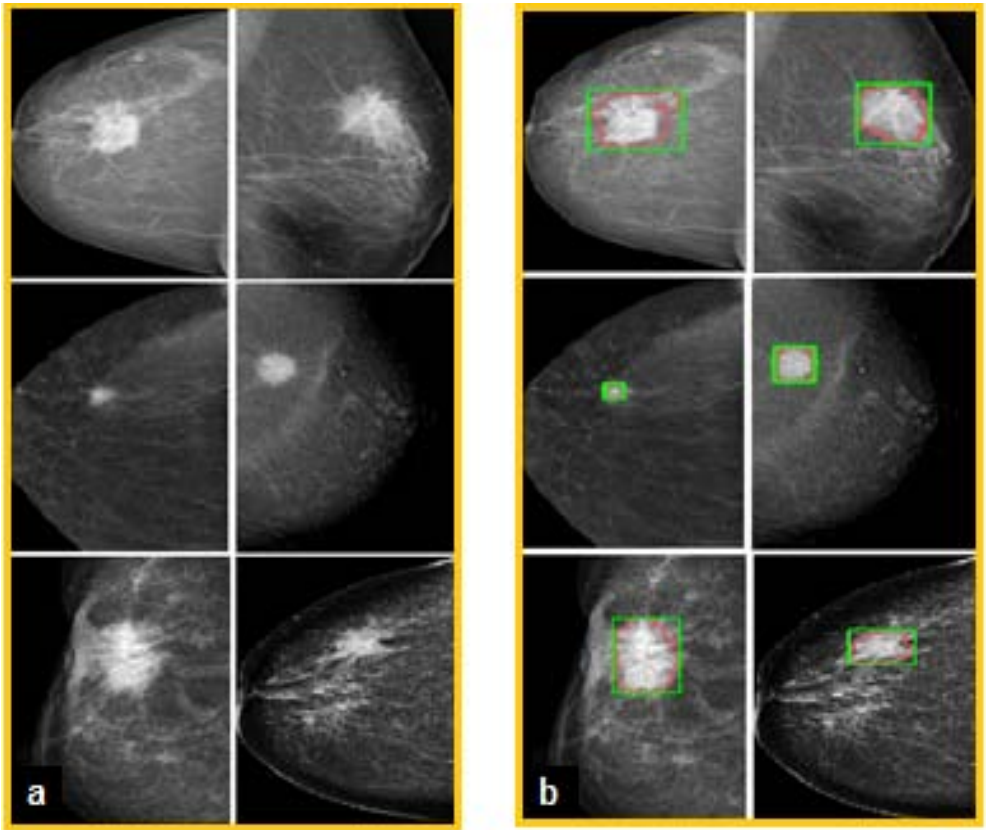

Fig. 8 Qualitative results of our speculated lesions detection approach: (a) original mammography image; (b) detection results by our method.

Table 3 Comparison with state-of-the-art methods.

\begin{tabular}{ll}
\hline Method & Classification rate \\
\hline Independent Component Analysis + Neural Networks [5] & $97.3 \%$ \\
Wavelet multi-resolution + Neural Networks [11] & $87.78 \%$ \\
Mathematical Morphology + Genetic Classifier [6] & $87 \%$ \\
Independent Component Analysis [8] & $79.3 \%$ \\
Directional Filtering + Gabor Wavelets + Bayes Classifier [9] & $74.4 \%$ \\
Skeleton Analysis [7] & $74 \%$ \\
SIFT Orientations + BDT Classifier (Our method) & $97.95 \%$ \\
\hline
\end{tabular}




\section{Conclusions}

In this paper, we present a novel system for stellate lesions detection in a digital mammogram, using a combination of multi-scale SIFT orientations and the well-known BDT classifier. The mammography image is firstly represented by a set of key points using DoG-based multi-scale approach [2]. The use of this approach allows us to process the low-resolution images directly and without any further processing, thus gaining a considerable amount of computation time. Then each key point is assigned an SIFT orientations to locally characterize it. Subsequently, we statistically compute three features for each pixel in the image mammogram based on these orientations, which will be finally fed into BDT to classify the corresponding pixel as normal or abnormal. We evaluate the performance of the proposed approach on mammography images from BCDR dataset. The detection results are quite satisfactory, which allow us to claim that it can be availed of to design an accurate computer-assisted diagnostic system. Ultimately, this study is conducted to establish the right path to anticipate the future evolution of image processing in medical field.

In our future work, we intend to extend the size of the training set by using mammography images from various medical databases and compare the obtained results with other spiculated lesions detection approaches.

\section{References}

[1] Berment, H., Becette, V., Mohallem, M., Ferreira, F., and Cherel, P. 2014. "Masses in Mammography: What Are the Underlying Anatomopathological Lesions?” Diagnostic and Interventional Imaging 95: 124-33.

[2] Lowe, D. G. 2001. "Object Recognition from Local Scale-Invariant Features.” In Proc. of the International Conference on Computer Vision.

[3] Liu, S., Babbs, C. F., and Delp, E. J. 1999. "Multiresolution Detection of Spiculated Lesions in Digital Mammograms.” School of Electrical and Computer Engineering.

[4] Karssemeijer, N., and Brake, G. M. T. E. 1996. "Detection of Stellate distortIons in Mammograms." IEEE Transactionson Medical Imaging 15 (5): 560-7.

[5] Ng, S. L., and Bishof, W. 1992. "Automated Detection and Classification of Breast Tumors.” Comput Biomed Res 25 (3): 218-37.

[6] Kegelmeyer, W. P., Pruneda, J. M., Bourland, P. D., Hillis, A., and Nipper, M. L. 1994. "Computer-Aided Mammographic Screening for Speculated Lesions.” Radiology 191: 331-6.

[7] Zhang, M., and Giger, M. L. 1996. "Mammographic Texture Analysis for the Detection of Spiculated Lesions.” Excerpta Medica 1119: 347-51.

[8] Campos, L. F. A., Silva, A., and Barros, A. K. 2005. "Diagnosis of Breast Cancer in Digital Mammograms Using Independent Component Analysis and Neural Networks.” In 10th Iberoamerican Congress on Pattern Recognition, CIARP 2005, Havana, Cuba, November 15-18, 2005.

[9] Ballerini, L., and Franzen, L. 2004. “Optimization of Morphological Filters with Applications in Breast Cancer Detection.” Applications of Evolutionary Computing, Evo Workshops.

[10] Kobatake, H., and Yoshinaga, Y. 1996. "Detection of Spicules on Mammogram Based on Skeleton Analysis." IEEE Transactions on Medical Imaging.

[11] Christoyianni, L., Koutras, A., Dermatas, E., and Kokkinakis, G. 2002. "Computer Aided Diagnosis of Breast Cancer in Digitized Mammograms." Computerised Medical Imaging and Graphics 26 (5): 309-19.

[12] Ferrari, R. J., Rangayyan, R. M., Desautels, J. E. L., and Frere, A. F. 2001. "Analysis of Asymmetry in Mammograms via Directional Filtering with Gabor Wavelets.” IEEE Transactions on Medical Imaging 20 (9): 953-64.

[13] Sampat, M. P., Markey, M. K., and Bovik, A. C. 2006. "Computer-Aided Detection and Diagnosis in Mammography.” Sharif University of Technology, School of Electrical Engineering, Medical Images Analysis and Processing(MIAP).

[14] Hwang, H. G., Choi, H. J., Lee, B. I., Yoon, H. K., Nam, S. H., and Choi, H. K. 2005. "Multiresolution Wavelet-Transformed Image Analysis of Histological Sections of Breast Carcinomas.” Cellular Oncology 27: 237-44.

[15] Sakellaropoulos, F., Skiadopoulos, S. G., Karahalieu, A. N., Costaridou, L., and Panayiotakis, G. 2006. "Using Wavelet-Based Features to Identify Masses in Dense Breast Parenchyma.” Digital Mammography, 8th International Workshop.

[16] Dippel, S., Stahl, M., Wiemker, R., and Blaffert, T. 2002. 
"Multiscale Contrast Enhancement for Radiographies: Laplacian Pyramid versus Fast Wavelet Transform.” IEEE Transactionson Medical Imaging 21 (4).

[17] Hassan, M., and Bermak, A. 2014. "Gas Classification Using Binary Decision Tree Classifier.” In IEEE International Symposium on Circuits and Systems.

[18] Guevara Lopez, M. A., Posada, N. G., Moura, D. C., Pollan, R. R., Franco Valiente, J. M., Ortega, C. S., et al. 2014. "BCDR: A Breast Cancer Digital Repository.” In 15th International Conference on Experimental Mechanics.

[19] Metz, C. E. 1989. "Some Practical Issues of Experimental Design and Data Analysis in Radiological ROC Studies.” Invest. Radiol. 24 (3): 234-45.

[20] Metz, C. E. 1996. "Evaluation of Digital Mammography by ROC Analysis." In Proceedings of the 3rd International Workshop on Digital Mammography, Chicago, Illinois, pp. 61-8. 